\title{
Die ubiquitäre allergologische Präsenz der Hauskatze
}

$\mathrm{D}$ ie Hauskatze (felis domesticus) ist rund um den Globus ein liebenswerter Hausgenosse. Zu ihren herausragenden Tugenden gehört ihre Putzsucht. Gerade diese aber trägt dazu bei, daß sie für ihre Gastfamilien ein hohes allergologisches Risiko birgt - und dies nicht nur für diese. Ihre Hauptallergene, Fel d 1 und Fel d 2, finden sich in den Hautanhangsdrüsen und im Katzenspeichel - und werden daher durch das Putzen über das gesamte Fell verbreitet.

Es hat sich gezeigt, daß Katzenalle rgene zu den häufigsten Innenrauma 1 le rgenen gehören. Das Risiko, sich zu sensibilisieren, besteht keineswegs ausschließlich bei unmittelbarem Katzenkontakt, da Katzenallergene nicht nur in Wohnungen von Katzenhaltern vorkommen, sondern auch in Gebäuden ohne Katzenhaltung. Dies bedeutet auch, daß Katzenallergiker selbst nach Abschaffung ihres Haustieres weiterhin von Katzenallergenen bedroht werden, nicht nur in der eigenen Wo h $\mathrm{n} \mathrm{ung}$, in der die Allergenkonzentration erst nach Monaten bis Jahren absinkt ( Wood et al., 1989), oder bei erneutem Katzenkontakt, sondern auch in Kind e rgärten, Schulen, öffentlichen Gebäuden oder sogar in Wohnungen, in denen niemals Katzen gehalten wurden (Munir et al., 1993; Bollinger et al., 1996; Custovic et al., 1996; Wahn et al., 1997).

Die Katzenallergene sind im Wohnungsstaub an kleinste Partikel ( $<5 \mu \mathrm{m}$ Durchmesser) gebunden; daher sind sie lungengängig und haben ausgezeichnete Schwebeeigenschaften, so daß sie durch Hausputz und Staubsaugen kaum eliminiert werden können. Im Gegenteil: Ihre Luftkonzentration wird durch das Staubsaugen erhöht (Luczynska et al., 1990). Kater verbreiten mehr Allergene als Katzen (Wentz et al., 1990). Nicht definitiv geklärt ist, welche Allerg e n k o n ze n tr a ti onen zu Sensibilisierungen und Sympto- men führen. Die von Gelber et al. (1993) vorgeschlagene Schwellenkonzentration von $8 \mu \mathrm{g}$ Fel d 1/g Staub dürfte zu hoch liegen. Eine Exposition gegen weniger als $0,5 \mu \mathrm{g}$ Fel d $1 / \mathrm{g}$ Staub ist in Wohnungen sensibilisierter Kinder aus Atopikerfamilien nachgewiesen worden (Munir et al., 1997). Derartige Konzentrationen werden in Gebäuden ohne Katzenhaltung häufig nachgewiesen. Wahn et al. (1997) haben hierzu eindrucksvoll Dosis-Wi rkungsrelationen für die kindliche Sensibilisierung in den ersten 3 Lebensjahren bei einer Fel d 1-Konzentration bereits $a b<25 \mathrm{ng} / \mathrm{g}$ Staub publiziert. Fel d 1 wurde von dieser Arbeitsgruppe in $85 \%$ aller Wohnungen nachgewiesen.

Sensibilisierungen gegen Katzena 11 e rgene sind häufig: Ca. 10-15\% der Patienten mit Asthma und/oder Rhinitis sind betroffen. Daß Tierallergien auch ohne Präsenz des betreffenden Tieres auftreten, hat der Linksunterzeichner vor mehr als 2 Jahrzehnten beschrieben (Schultze-Werninghaus et al., 1976); dieser Befund wurde in neuerer Zeit bestätigt (Braso-Aznar et al., 1995).

Die weite Verbreitung der Katzena 11 e rgene bedeutet, daß unsere Therapiekonzepte bei Katzenallergie überprüft werden müssen. Bislang gilt: Bei $\mathrm{K}$ a t $z$ e n all e rgie ist die Abschaffung des Tieres zur Vermeidung der Allerg e n e xposition ausreichend. Eine Spezifische Immuntherapie wird in den Empfehlungen aller Fachgremien sehr zurückhaltend behandelt. Damit wird aber möglicherweise auf eine wichtige Therapieoption verzichtet, die als einzige

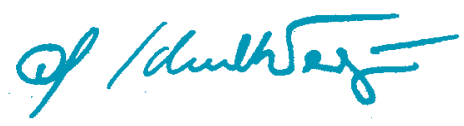

Prof. Dr. G. Schultze-Werninghaus zu einer Verminderung der Reaktionsbereitschaft von Katzenallergikern bei K a t z e n a ll e rgenkontakt führen könnte.

Die 7 vorliegenden Studien über die Erfolge einer Spezifischen Immuntherapie bei Katzenallergie sind positiv ausgefallen, die Langzeitwirkung hält über Jahre an (WHO-Positionspapier 1998, Varney et al., 1997). Dennoch empfiehlt die WHO primär eine Katzenkarenz und eine Immuntherapie nur dann, wenn die Karenz nicht ausreichend wirksam ist bzw. der Katzenkontakt Zuhause oder am Arbeitsplatz weiterbesteht.

Neue Wege einer Immuntherapie bei Katzenallergie werden gesucht. Die $\mathrm{K}$ a t $\mathrm{z}$ e n a ll e rgie ist als Modell für neue Formen der Immuntherapie besonders geeignet, da die Symptomatik ganz wesentlich durch ein einziges Protein, Fel d 1, verursacht wird. Erste positive Studien mit T-Zell-reaktiven Fel d 1Peptiden zur Induktion einer T-ZellToleranz sind publiziert worden (Normal et al., 1996; Pène et al., 1998). Diese Entwicklung wird die Spezifische Immuntherapie bei Katzenallerg i e sicherlich begünstigen.

Fazit: Das Katzenallergen ist weit verbreitet - die Katzenallergie ist keineswegs an die Präsenz des Tieres gebunden. Die Fachgesellschaften sind daher aufgefordert, ihre Empfehlungen zur Immuntherapie bei Katzenallerg i e zu überprüfen.

Für das Jahr 1999 wünschen Ihnen die Herausgeber Gesundheit, Glück und Erfolg!

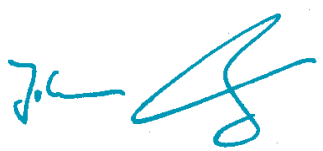

Prof. Dr. Dr. J. Ring 